\title{
Self-Aligned Silicidation of Surround Gate Vertical MOSFETs for Low Cost RF Applications
}

\author{
M. M. A. Hakim, L. Tan, A. Abuelgasim, K. Mallik, Member, IEEE, S. Connor, A. Bousquet, \\ C. H. de Groot, Senior Member, IEEE, W. Redman-White, Senior Member, IEEE, \\ S. Hall, Senior Member, IEEE, and P. Ashburn, Member, IEEE
}

\begin{abstract}
We report for the first time a CMOS-compatible silicidation technology for surround-gate vertical MOSFETs. The technology uses a double spacer comprising a polysilicon spacer for the surround gate and a nitride spacer for silicidation and is successfully integrated with a Fillet Local OXidation (FILOX) process, which thereby delivers low overlap capacitance and highdrive-current vertical devices. Silicided 80 -nm vertical $n$-channel devices fabricated using $0.5-\mu \mathrm{m}$ lithography are compared with nonsilicided devices. A source-drain (S/D) activation anneal of $30 \mathrm{~s}$ at $1100{ }^{\circ} \mathrm{C}$ is shown to deliver a channel length of $80 \mathrm{~nm}$, and the silicidation gives a $60 \%$ improvement in drive current in comparison with nonsilicided devices. The silicided devices exhibit a subthreshold slope $(\mathrm{S})$ of $\mathbf{8 7} \mathrm{mV} / \mathrm{dec}$ and a drain-induced barrier lowering (DIBL) of $80 \mathrm{mV} / \mathrm{V}$, compared with $86 \mathrm{mV} / \mathrm{dec}$ and $60 \mathrm{mV} / \mathrm{V}$ for nonsilicided devices. S-parameter measurements on the 80-nm vertical nMOS devices give an $f_{T}$ of $20 \mathrm{GHz}$, which is approximately two times higher than expected for comparable lateral MOSFETs fabricated using the same $0.5-\mu \mathrm{m}$ lithography. Issues associated with silicidation down the pillar sidewall are investigated by reducing the activation anneal time to bring the silicided region closer to the p-n junction at the top of the pillar. In this situation, nonlinear transistor turn-on is observed in drain-on-top operation and dramatically degraded drive current in source-on-top operation. This behavior is interpreted using mixed-mode simulations, which show that a Schottky contact is formed around the perimeter of the pillar when the silicided contact penetrates too close to the top S/D junction down the side of the pillar.
\end{abstract}

Index Terms-Fillet Local OXidation (FILOX), interface states, silicidation, vertical MOSFETs (v-MOSFETs).

\section{INTRODUCTION}

$\mathbf{T}$ HIN-PILLAR fully depleted surround-gate vertical MOSFETs (v-MOSFETs) are being researched as candidates for end-of-roadmap CMOS technology [1]-[4]. The advantages of these devices stem from their excellent

Manuscript received March 19, 2010; revised July 20, 2010; accepted September 17, 2010. Date of publication October 25, 2010; date of current version November 19, 2010. This work was supported by the Engineering and Physical Sciences Research Council. The review of this paper was arranged by Editor H. S. Momose.

M. M. A. Hakim, A. Abuelgasim, K. Mallik, C. H. de Groot, W. Redman-White, and P. Ashburn are with the School of Electronics and Computer Science, University of Southampton, SO17 1BJ Southampton, U.K. (e-mail: mmah@ecs.soton.ac.uk).

L. Tan and S. Hall are with the Department of Electrical Engineering and Electronics, University of Liverpool, L69 3GJ Liverpool, U.K.

S. Connor and A. Bousquet are with Plessey Semiconductors Ltd., SN2 2QW Swindon, U.K.

Color versions of one or more of the figures in this paper are available online at http://ieeexplore.iee.org.

Digital Object Identifier 10.1109/TED.2010.2082293 electrostatic control, the resulting short-channel effect (SCE) immunity, and high drive current. Better SCE immunity also allows the use of low-doped/undoped channels, which improves carrier transport properties (mobility) and reduces dopant fluctuation problems. Scaling the pillar thickness in the fully depleted regime has been shown to deliver excellent subthreshold and drain-induced barrier lowering (DIBL) characteristics, although the expected improvement in drive current has only been demonstrated for pillar diameters of less than $20 \mathrm{~nm}$, where a very strong volume inversion exists in the channel [1], [2]. However, these devices usually require aggressive electron beam lithography and/or complex processing [1]-[4], and so far, no silicidation technology has been reported for these devices.

Thick-pillar surround-gate v-MOSFETs are also of interest [5]-[17] because they can easily be integrated into a mature CMOS technology to provide a low-cost route to radio-frequency (RF) transistors. Thick-pillar surround-gate v-MOSFETs have several obvious advantages over planar MOSFETs. First, surround-gate structures allow more channel width and drive current per unit silicon area. Second, the channel length is controlled by nonlithographic methods, allowing the devices with sub-100-nm channel length to be realized without any advanced lithography. Hence, the advantages of short-channel transistors can be enjoyed without costly processing. Third, co-integration of surround-gate v-MOSFETs and conventional planar MOSFETs can easily be achieved due to the bulk silicon starting material. However, v-MOSFETs have several important disadvantages, i.e., high overlap capacitance, dry etch damage on the pillar sidewall, and lack of an appropriate silicidation technology. The problem of overlap capacitance has been addressed using Fillet Local OXidation (FILOX) [15], [16]. In this process, a thicker oxide is grown at the bottom and top of the active pillar using nitride spacer to suppress oxidation on the pillar sidewall. The thick FILOX oxide reduces the overlap capacitance between the gate and the source/drain (S/D) electrodes. Dry etch damage occurs during pillar dry etch and polysilicon gate etch, and degrades the subthreshold slope. Damage occurring during pillar dry etch can be eliminated by sacrificial oxidation, and we have recently shown how the device architecture and the FILOX process [15] can be optimized to eliminate dry etch damage during silicon gate etch and deliver excellent values of subthreshold slope [18], [19]. However, to date, there have been no reports in the literature on silicidation technologies for surround-gate $\mathrm{v}$-MOSFETs. Self-aligned silicidation is one of the key features 
of advanced MOSFETs, which reduces S/D series resistances and increases the drive current, and hence is essential for RF applications.

We recently reported preliminary results on silicided v-MOSFETs [20] and showed that a subthreshold slope degradation and drive current asymmetry in silicided devices could be eliminated by using a frame-gate architecture [18]. In this approach, a frame of polysilicon is incorporated around the perimeter of the pillar, which unfortunately has the disadvantage of increasing the overlap capacitance. In this paper, we show that excellent values of subthreshold slope can be achieved in silicided devices without resorting to a frame gate. A double spacer is implemented, comprising a polysilicon spacer for the surround gate and a nitride spacer for the silicidation, and excellent values of subthreshold slope are achieved by optimizing the FILOX process and S/D anneal. The silicided 80 -nm n-channel devices deliver a $60 \%$ improvement in drive current in comparison to nonsilicided devices, an excellent subthreshold slope of $87 \mathrm{mV} / \mathrm{dec}$, and a DIBL of $80 \mathrm{mV} / \mathrm{V}$. The RF performance of the v-MOSFETs is characterized, and issues with the silicidation down the pillar sidewall are investigated.

\section{EXPERIMENTAL PROCEDURE}

A mature $0.5-\mu \mathrm{m}$ CMOS technology was used to fabricate v-MOSFETs. Boron-doped $(0.75-1.25 \Omega \cdot \mathrm{cm})(100)$ wafers were taken as the starting material, and a p-type body was formed by boron implantation $\left(2 \times 10^{14} / \mathrm{cm}^{2}, 100 \mathrm{keV}, 7^{\circ}\right.$ tilt) and high-temperature drive-in. Transistors with different channel lengths were then produced by varying the Si pillar dry etch, with pillar heights varying from 300 to $450 \mathrm{~nm}$. A sacrificial oxidation was performed to eliminate dry etch damage and reduce the surface roughness on the pillar sidewall. After stripping of this oxide, a 10-nm stress relief oxide was thermally grown at $900{ }^{\circ} \mathrm{C}$. For the FILOX process [15], a 90-nm silicon nitride was deposited at $720{ }^{\circ} \mathrm{C}$ and anisotropically etched to create a nitride spacer; subsequently, a 60-nm FILOX oxide layer was thermally grown at $1100{ }^{\circ} \mathrm{C}$ [Fig. 1(a)].

The source and drain were then implanted using a single $3 \times 10^{15} \mathrm{~cm}^{-2} 110-\mathrm{keV}$ As implant at $7^{\circ}$ tilt, and the nitride fillet and pad oxide were subsequently removed by wet etch. A 2.8-nm gate oxide was then grown at $700{ }^{\circ} \mathrm{C}$, and a $230-\mathrm{nm}$ in situ phosphorous-doped $\left(1 \times 10^{20} / \mathrm{cm}^{3}\right)$ polysilicon gate was deposited and patterned by dry etch to create a surround gate. A rapid thermal annealing (RTA) at $1100{ }^{\circ} \mathrm{C}$ for 10 or $30 \mathrm{~s}$ was then performed for dopant activation, which resulted in different junction depths and, hence, different channel lengths for the same pillar height. In the silicided wafers, the underlying FILOX oxide was also removed by dry etch just after the gate etch. A 20-nm oxide layer and an 80-nm nitride layer were then deposited, and subsequently, the nitride layer was dry etched to leave nitride spacers over the polysilicon fillet around the pillar sidewall [Fig. 1(b)].

After a brief hydrofluoric acid (HF) dip etch, a 20-nm Ni layer was deposited by e-beam evaporation. A silicide layer was formed by annealing for $30 \mathrm{~s}$ at $450{ }^{\circ} \mathrm{C}$, and unreacted $\mathrm{Ni}$ was removed using a piranha solution. In this way, the $\mathrm{S} / \mathrm{D}$ regions and the horizontal portions of the polysilicon gate

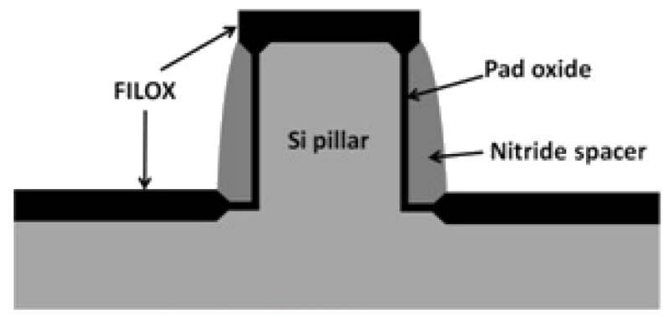

(a)

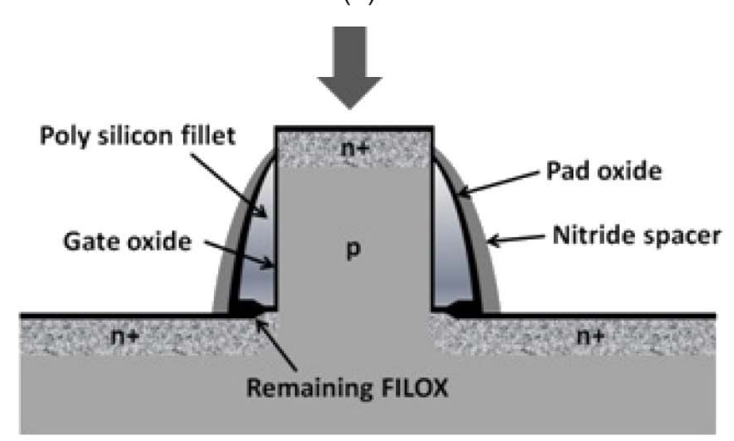

(b)

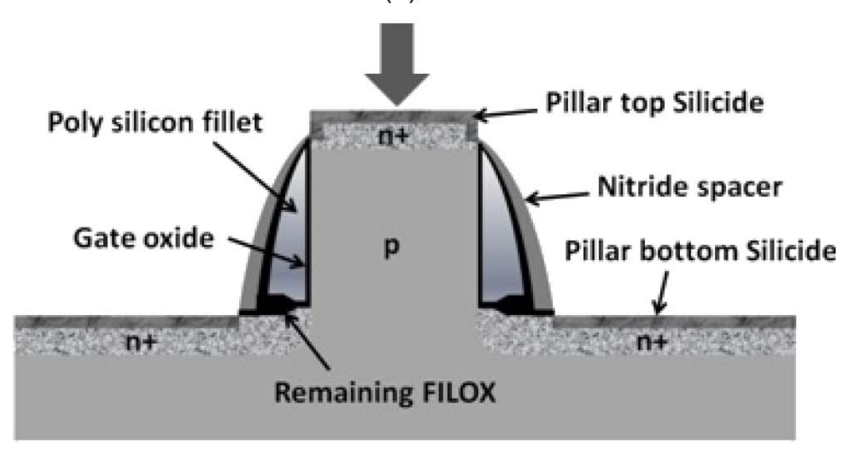

(c)

Fig. 1. Schematic process flow for silicidation of surround-gate v-MOSFETs. (a) FILOX process [15], [16]. (b) Nitride spacer process for silicidation. (c) Silicided v-MOSFETs.

were silicided [Fig. 1(c)]. A 300-nm LTO layer was used as a passivation layer, and contacts and metal were defined.

The gate oxide thickness was measured from the second derivative of the high-frequency capacitance/voltage characteristic [21], and a value of $2.8 \mathrm{~nm}$ was obtained. The body doping was also extracted from this measurement, and a value of $1 \times 10^{18} / \mathrm{cm}^{3}$ was obtained. A similar method was used to measure the FILOX oxide thickness at the end of the process, and a value in the range of $35-40 \mathrm{~nm}$ was obtained. S/D sheet resistances were measured from Van der Pauw structures; values of 32 and $42 \Omega /$ sq were obtained for 30- and 10-s anneals in nonsilicided wafers, and $7 \Omega /$ sq were obtained in silicided wafers. The channel length $L$ was obtained from measurements of the pillar heights using cross-sectional scanning electron microscope (SEM) and measurements of the junction depths by stain etching in a $\mathrm{HF}: \mathrm{HNO}_{3}: \mathrm{CH}_{3} \mathrm{COOH}, 1: 3: 8$ for $5-10 \mathrm{~s}$ [22]. For a 10 -s RTA at $1100{ }^{\circ} \mathrm{C}$, channel lengths of 120, 170, and $220 \mathrm{~nm}$ were obtained for pillar heights of 350, 400, and $450 \mathrm{~nm}$, respectively. For a 30-s RTA at $1100{ }^{\circ} \mathrm{C}$, a channel length of $80 \mathrm{~nm}$ was obtained. We also extracted the channel length by gate-channel capacitance $C_{\mathrm{GC}}$ measurements [23] and the drain conductance method [24]. The 

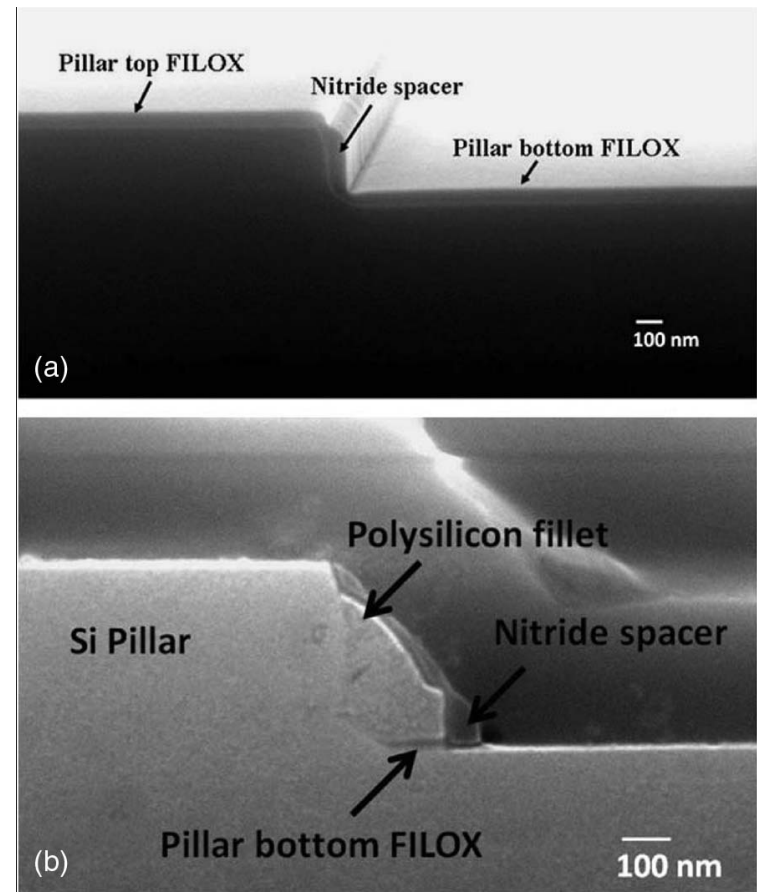

Fig. 2. SEM cross section of the v-MOSFET process (a) directly after the FILOX [15], [16] oxidation and (b) after the nitride spacer process prior to silicidation.

extracted channel lengths by these measurements were in good agreement with the preceding results. Both dc and ac transistor characterizations were performed from on-wafer measurements using a Cascade semiautomatic Summit 12000B-AP probe station. DC measurements were performed using an Agilent B1500A semiconductor device analyzer, and ac electrical data was acquired from S-parameter measurements using an Agilent 8361 A microwave vector network analyzer. The parasitics were de-embedded from the S-parameter measurements using open, short, load, and through structures.

\section{RESULTS}

\section{A. Process Characterization}

A SEM cross section of the transistor immediately after the FILOX process is shown in Fig. 2(a). The figure clearly shows the 70-nm nitride spacer and the presence of the FILOX oxide at the top and bottom of the pillar. The thickness of the FILOX oxide is $60 \mathrm{~nm}$, in agreement with expectations. Oxide encroachment of $75 \mathrm{~nm}$ can be seen at the pillar top, but there is no such encroachment at the pillar bottom. Fig. 2(b) shows a SEM micrograph after the formation of the nitride spacer on the polysilicon surround gate ready for silicidation. The successful processing of the nitride spacer on the polysilicon gate can be clearly seen. The nitride spacer and polysilicon gate have thicknesses of 70 and $200 \mathrm{~nm}$, respectively, at the pillar bottom.

Fig. 3(a) shows a SEM cross section of a 120-nm v-MOSFET after silicide formation. A 45-nm-thick continuous silicide layer can be easily seen at the pillar top and bottom. The silicided surface is rather rough due to the polycrystalline grains formed during the silicidation process. Furthermore, silicidation down the pillar sidewall can be observed, which is found to be around

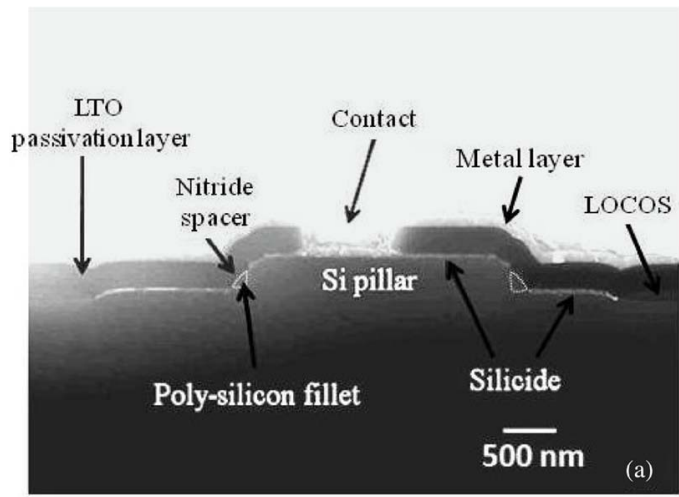

LTO

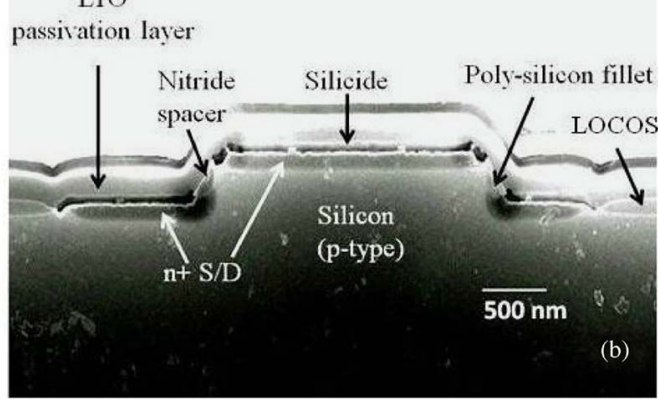

Fig. 3. SEM cross section of a 120-nm v-MOSFET after silicide formation (a) without any etch and (b) after stain etching [22]. The outline of the sidewall polysilicon fillet has been highlighted in (a) for clarity.

$190 \mathrm{~nm}$ down from the pillar top. Fig. 3(b) shows the SEM cross section at the same stage of the process but after a delineation etch [22] that has been used to stain the p-n junction. The silicided regions and the S/D junctions can be easily seen from this image, although the nickel silicide has been etched by the $\mathrm{HF}$ in the stain etch. The junction depth is around $200 \mathrm{~nm}$, and from the 190-nm extension of the silicide layer down the pillar sidewall, which was measured from Fig. 3(a), the silicided region extends to within 5-15 nm of the top p-n junction. At the pillar bottom, the silicide layer is $250-270 \mathrm{~nm}$ away from the bottom p-n junction in the horizontal direction. Similar experiments on 80-nm v-MOSFETS gave a top p-n junction depth of $240 \mathrm{~nm}$ and extension of the silicide layer to within $45-55 \mathrm{~nm}$ of the top p-n junction.

\section{B. DC Electrical Characteristics}

Fig. 4 shows typical output characteristics of $80-\mathrm{nm}$ v-MOSFETs for drain-on-top (DOT) and source-on-top (SOT) modes of operation. Results are shown for (dashed line) silicided and (solid line) nonsilicided v-MOSFETs. For the DOT mode of operation, Fig. 4(a) shows a drive current of $400 \mu \mathrm{A} / \mu \mathrm{m}$ for silicided devices at a gate voltage overdrive of $1 \mathrm{~V}$ and a $V_{D S}$ of $1.5 \mathrm{~V}$, which compares with a value of $250 \mu \mathrm{A} / \mu \mathrm{m}$ for nonsilicided devices. The silicidation has therefore delivered a $60 \%$ improvement in drive current for the DOT mode of operation. Fig. 4(b) shows the equivalent results for the SOT mode of operation, and drive currents of 385 and $255 \mu \mathrm{A} / \mu \mathrm{m}$ are obtained for silicided and nonsilicided devices, respectively. For SOT operation, silicidation has delivered a $51 \%$ improvement in drive current. Measurements were made 


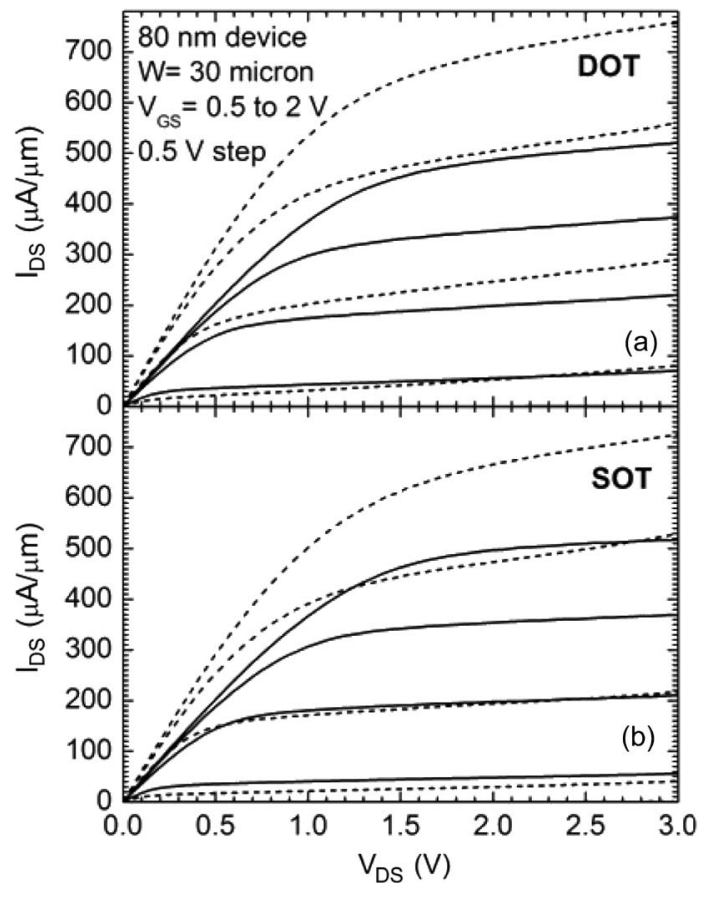

Fig. 4. Output characteristics of 80-nm v-MOSFETs annealed for $30 \mathrm{~s}$ at $1100{ }^{\circ} \mathrm{C}$ for (a) DOT and (b) SOT modes of operation. Results are shown for (dashed line) silicided and (solid line) nonsilicided v-MOSFETs.

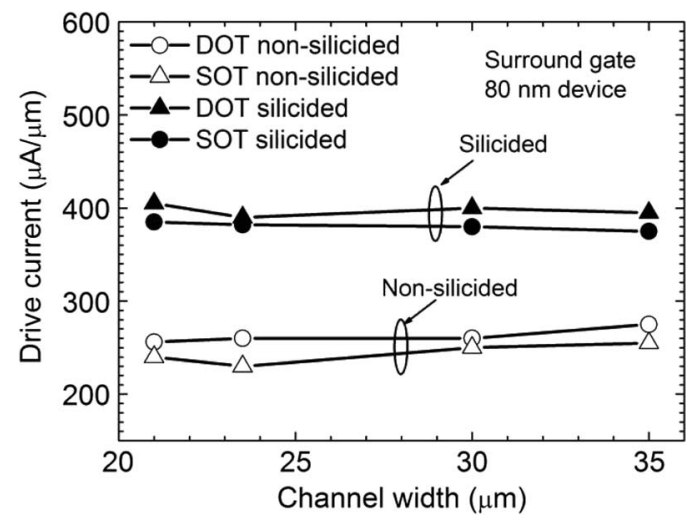

Fig. 5. Drive current (i.e., for a gate voltage overdrive of $1 \mathrm{~V}$ and a $V_{D S}$ of $1.5 \mathrm{~V}$ ) of 80 -nm surround-gate v-MOSFETs for different drawn channel widths. Results are shown for silicided and nonsilicided v-MOSFETs and for DOT and SOT modes of operation.

on v-MOSFETs with a variety of different channel widths, and a similar behavior was observed (Fig. 5). For example, for v-MOSFETs with a channel width of $21 \mu \mathrm{m}$, silicidation gave $65 \%$ and $51 \%$ improvements in the drive current for DOT and SOT modes of operation, respectively. This result indicates that the characteristics of these v-MOSFETs are rather symmetrical.

Fig. 6 shows the effects of silicidation on the subthreshold characteristics of 80 -nm v-MOSFETs. The silicided and nonsilicided devices exhibit almost identical values of subthreshold slope, with values of 87 and $86 \mathrm{mV} / \mathrm{dec}$, respectively. However, there are small differences in the values of DIBL ( 80 and $60 \mathrm{mV} / \mathrm{V})$ and threshold voltage $(0.34$ and $0.23 \mathrm{~V})$ for silicided and nonsilicided devices, respectively. Measurements on 120-nm v-MOSFETs (not shown) gave a smaller degradation of the subthreshold slope after silicidation from 78 to $85 \mathrm{mV} / \mathrm{dec}$. The 120-nm v-MOSFETS also showed a $0.17-\mathrm{V}$ increase in

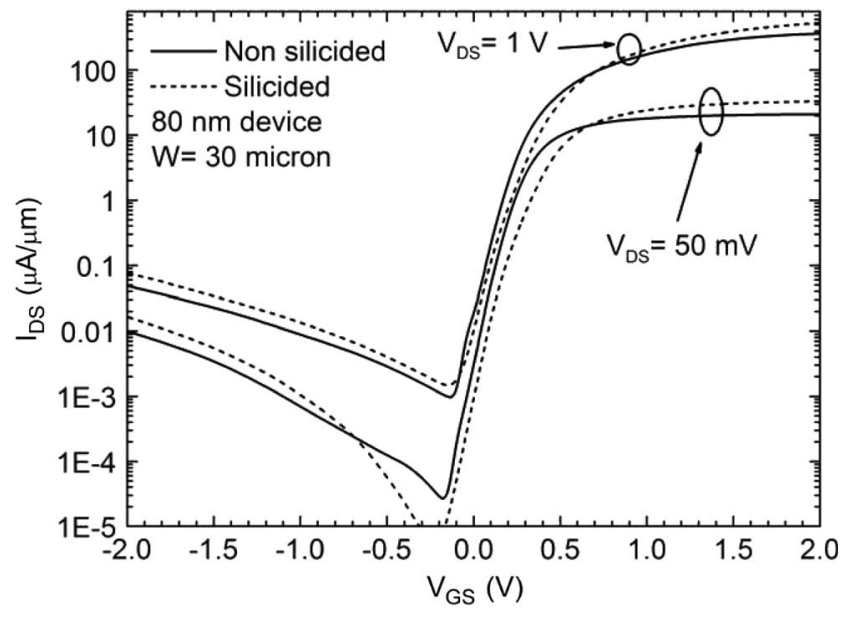

Fig. 6. Subthreshold characteristics of $80-\mathrm{nm}$ v-MOSFETs annealed for $30 \mathrm{~s}$ at $1100^{\circ} \mathrm{C}$. Results are shown for silicided and nonsilicided v-MOSFETs.

the threshold voltage after silicidation. The OFF-state leakage for both silicided and nonsilicided devices increases gradually with increasing negative gate bias in Fig. 6 due to gate-induced drain leakage and gate leakage. Measurements have shown that the gate leakage originates from the bottom of the pillar and is presumably due to the thinning of the gate oxide at the bottom corner. We have seen much lower values of OFF-state leakage in some of our FILOX v-MOSFETs [18], [19], indicating that the leakage current can be significantly reduced by optimizing the FILOX process. For example, a thinner nitride spacer will allow the FILOX oxide to encroach further around the bottom of the pillar, thereby thickening the oxide at the bottom corner.

To investigate the effects of silicidation down the pillar sidewall, Fig. 7 shows the output characteristics of transistors (120-nm channel length), which have been given a shorter anneal of $10 \mathrm{~s}$ at $1100^{\circ} \mathrm{C}$. For DOT operation, Fig. 7(a) shows drive currents for a gate voltage overdrive of $1 \mathrm{~V}$ and a $V_{D S}$ of $1.5 \mathrm{~V}$ of 240 and $180 \mu \mathrm{A} / \mu \mathrm{m}$ for silicided and nonsilicided transistors, respectively, indicating a $30 \%$ improvement. However, below $V_{D S}=1 \mathrm{~V}$, a nonlinear transistor turn-on can be seen in the characteristic of the silicided device. For SOT operation, Fig. 7(b) shows that the silicided transistors surprisingly have a much lower drive current than the nonsilicided transistors, with values of 55 and $170 \mu \mathrm{A} / \mu \mathrm{m}$, respectively.

\section{AC Electrical Characteristics}

Fig. 8 shows the gate-S/D capacitances $C_{\mathrm{GS} / \mathrm{D}}$ at the pillar top and pillar bottom as a function of gate voltage. Measurements were made on test structures with an array of 500 transistors connected in parallel, and the parasitic interconnect capacitances were decoupled by measurements on dummy metal line structures. The measured capacitances in accumulation represent overlap capacitances, whereas measured capacitances in inversion represent the sum of overlap and gatechannel capacitances. It is observed that FILOX vertical transistors exhibit pillar top and pillar bottom overlap capacitances $C_{\mathrm{GS} / \mathrm{D}}$ of 1.1 and $2.25 \mathrm{fF} / \mu \mathrm{m}$, respectively. For a $0.5-\mu \mathrm{m}$ technology node, planar MOSFETs have an overlap capacitance around $1 \mathrm{fF} / \mu \mathrm{m}$ when estimated for a gate oxide thickness of 


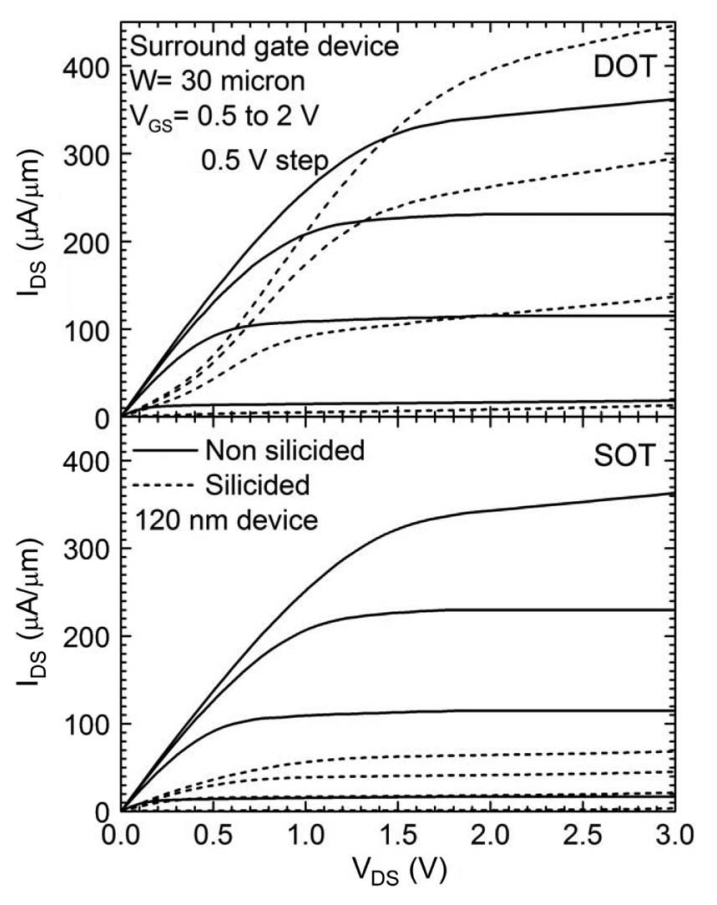

Fig. 7. Output characteristics of 120-nm surround-gate v-MOSFETs annealed for $10 \mathrm{~s}$ at $1100{ }^{\circ} \mathrm{C}$ for DOT and SOT modes of operation. Results are shown for silicided and nonsilicided v-MOSFETs.

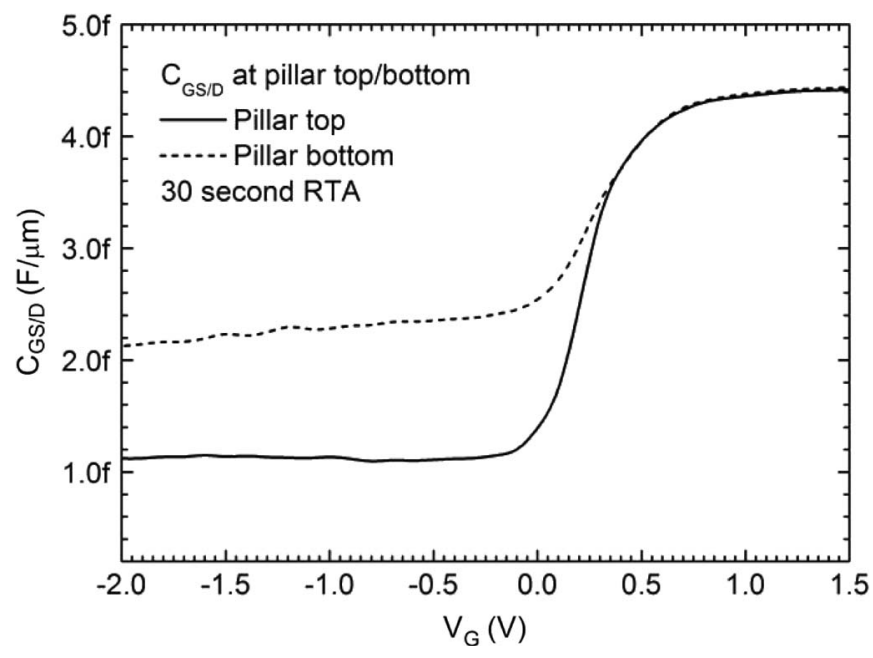

Fig. 8. Gate-S/D capacitances $C_{\mathrm{GS} / \mathrm{D}}$ at the pillar top and bottom as a function of gate voltage for surround-gate FILOX v-MOSFETs. These devices have a channel length of $80 \mathrm{~nm}$ and were annealed for $30 \mathrm{~s}$ at $1100{ }^{\circ} \mathrm{C}$.

$2.8 \mathrm{~nm}$ [25]. This indicates that FILOX v-MOSFETs exhibit a similar value of overlap capacitance at the top of the pillar to comparable planar MOSFETs. This is achieved by using a thick FILOX oxide and a significant polysilicon fillet overetch $(\sim 170 \mathrm{~nm}$ from the pillar top as calculated from Fig. 3), thereby reducing the overlap between the polysilicon gate and the top p-n junction. However, the pillar bottom overlap capacitance is twice that of a comparable $0.5-\mu \mathrm{m}$ planar MOSFET. This is due to the use of a thick polysilicon surround gate $(\sim 200 \mathrm{~nm})$ that creates a large overlap at the pillar bottom. The pillar bottom overlap capacitance can be easily optimized by reducing the polysilicon gate thickness, increasing the FILOX thickness, or reducing the FILOX nitride spacer thickness.

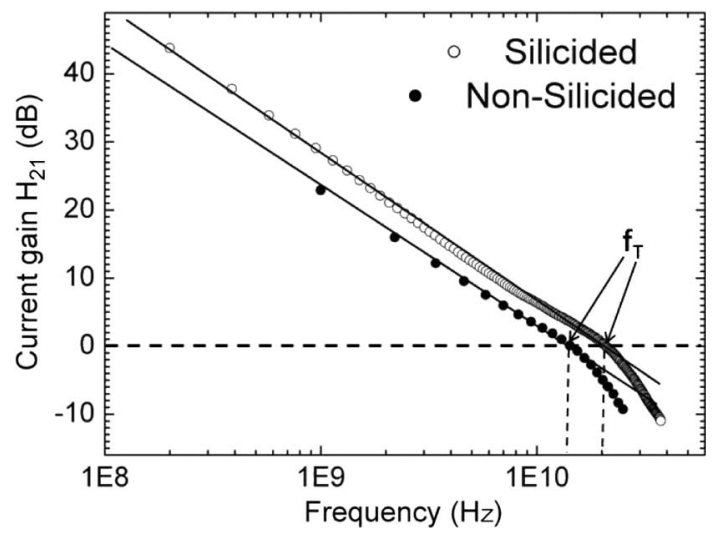

Fig. 9. Current gain as a function of frequency for surround-gate FILOX v-MOSFETs with and without silicidation. The silicided v-MOSFETs have a channel length of $80 \mathrm{~nm}$, and the nonsilicided v-MOSFETs have a channel length of $70 \mathrm{~nm}$. The $20-\mathrm{dB} / \mathrm{dec}$ lines are shown for illustration. The devices had a channel width of $30 \mu \mathrm{m}$, and the measurements were made at biases of $V_{G S}=0.75 \mathrm{~V}$ and $V_{D S}=1 \mathrm{~V}$.

Fig. 9 shows the current gain as a function of frequency for silicided and nonsilicided v-MOSFETs. For the silicided v-MOSFET, a cutoff frequency $f_{T}$ of around $20 \mathrm{GHz}$ has been achieved, which compares with a value of $14.6 \mathrm{GHz}$ for the nonsilicided v-MOSFET. This 6-GHz improvement in $f_{T}$ has been achieved by reducing the S/D series resistances using the aforementioned silicidation process. It should be noted that this improvement in $f_{T}$ has been achieved, even though the silicided v-MOSFET has a slightly longer measured channel length $(80 \mathrm{~nm})$ than the nonsilicided v-MOSFET $(70 \mathrm{~nm})$.

\section{DISCUSSION}

The preceding results have shown that a silicidation technology has been successfully developed for 80-nm v-MOSFETs. The silicidation gives a $60 \%$ improvement in drive current, and the output characteristics are symmetrical in SOT and DOT modes of operation. A drive current of $400 \mu \mathrm{A} / \mu \mathrm{m}$ has been achieved, which is significantly higher than previous results reported in the literature, as shown in Table I [5]. Furthermore, the AC results in Fig. 9 show that an $f_{T}$ of $20 \mathrm{GHz}$ has been achieved in the silicided 80-nm v-MOSFETs, which represents an excellent outcome for a technology that only used $0.5-\mu \mathrm{m}$ lithography. To investigate how this performance compares with that achieved for conventional lateral MOSFETs at the same $0.5-\mu \mathrm{m}$ lithography node, Fig. 10 plots $f_{T}$ as a function of technology node for a variety of conventional planar RF technologies taken from [26]. It can be seen that our 80-nm silicided v-MOSFET delivers a significantly higher $f_{T}$ than comparable planar MOSFET technologies implemented using the same $0.5-\mu \mathrm{m}$ lithography. We have also estimated the peak transconductances $G_{M}$ of silicided devices from Fig. 6, and these were found to be $1.4 \times 10^{-3}$ and $1.2 \times 10^{-2} \mathrm{~A} / \mathrm{V}$ for $V_{D S}=0.05$ and $1 \mathrm{~V}$, respectively. These $G_{M}$ values are noticeably better than those reported for planar MOSFETs with comparable 0.5/0.7- $\mu \mathrm{m}$ channel lengths [27], [28] and better than the $0.18-\mu \mathrm{m}$ planar MOSFET reported in [29]. It can therefore be concluded that $\mathrm{v}$-MOSFETs offer a viable route for improving the RF performance of mature lateral CMOS 
TABLE I

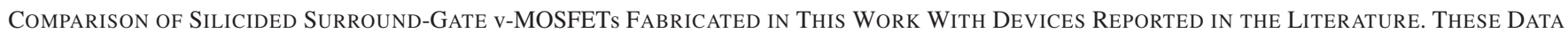
Were TaKen From [5] AND Updated With More Recent Results. The Values of $I_{\text {on }}$ Were Calculated For $V_{D} S=V_{D} D$ AND For a 1 -V Gate Overdrive. To Ensure a Meaningful Comparison, Fully Depleted Thin-Pillar v-MOSFETs Have BeEn Excluded From the TABle, Because Improved Drive Would Be EXPeCted From These Devices Due to the Volume InVERsion From the ACTION OF THE DUAL OR SURROUND GATES

\begin{tabular}{|c|c|c|c|c|c|c|c|}
\hline Parameter & $\begin{array}{c}\mathrm{L} \\
(\mathrm{nm})\end{array}$ & $\begin{array}{r}t_{o x} \\
(n m)\end{array}$ & $\begin{array}{c}N_{A} \\
\left(10^{17} / c c\right)\end{array}$ & $\begin{array}{l}V_{D D} \\
\text { (V) }\end{array}$ & $\begin{array}{c}\text { lon } \\
(\mu \mathrm{A} / \mu \mathrm{m})\end{array}$ & $\begin{array}{c}\mathrm{S} \\
\text { (mV/dec) }\end{array}$ & $\begin{array}{c}\text { DIBL } \\
(\mathrm{mV} / \mathrm{V})\end{array}$ \\
\hline Schulz et al [5] & 100 & 3 & 20 & 1.5 & 240 & 102 & 70 \\
\hline Schulz et al [5] & 50 & 3 & 70 & 1.5 & 80 & 166 & 300 \\
\hline VRG [13] & 100 & 2.8 & 35 & 1.5 & 140 & 90 & 30 \\
\hline VRG [13] & 50 & 2.8 & 35 & 1.5 & 100 & 105 & 90 \\
\hline Mori et al [11] & 100 & 7 & 20 & 1.5 & 160 & 100 & 73 \\
\hline Gili et al [16] & 125 & 3 & 40 & 1.5 & 127 & 107 & 80 \\
\hline Previous report [18] & 100 & 2.6 & 10 & 1.5 & 160 & $70-80$ & $30-35$ \\
\hline $\begin{array}{l}\text { Surround gate (non- } \\
\text { silicided) }\end{array}$ & 120 & 2.8 & 10 & 1.5 & 180 & 78 & 20 \\
\hline Surround gate (Silicided) & 120 & 2.8 & 10 & 1.5 & 240 & 85 & 40 \\
\hline $\begin{array}{l}\text { Surround gate (non- } \\
\text { silicided) }\end{array}$ & 80 & 2.8 & 10 & 1.5 & 250 & 86 & 60 \\
\hline Surround gate (Silicided) & 80 & 2.8 & 10 & 1.5 & 400 & 87 & 80 \\
\hline
\end{tabular}

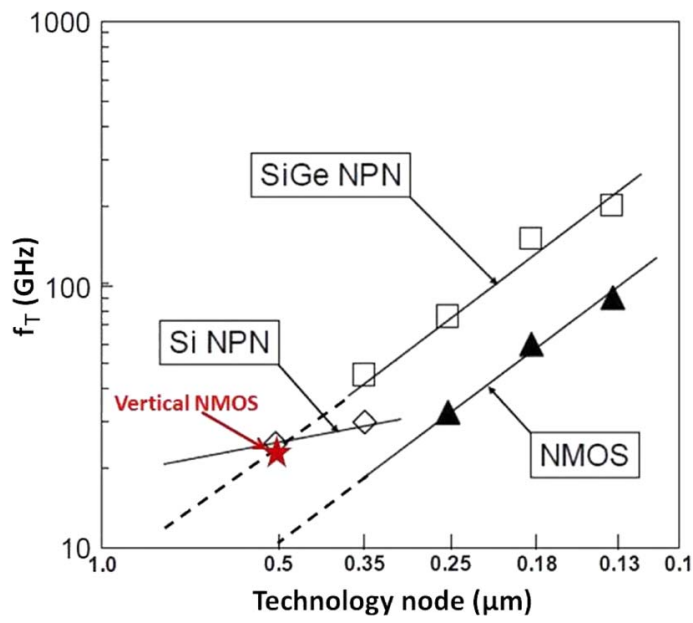

Fig. 10. Cut-off frequency $f_{T}$ as a function of technology node for the silicided 80-nm surround-gate FILOX v-MOSFET and a variety of comparator technologies taken from [26].

technologies at the cost of only one additional mask for pillar dry etch.

The results in Fig. 7 show a dramatic degradation in the dc performance of the silicided v-MOSFETs when the anneal time is reduced to bring the silicided region closer to the top S/D junction. As previously discussed, the 30-s anneal used for the 80-nm v-MOSFETs locates the silicide layer $45-55 \mathrm{~nm}$ from the top S/D junction, whereas the 10-s anneal used for the 120-nm v-MOSFETs reduces this distance to 5-15 $\mathrm{nm}$. SIMS analysis on the v-MOSFET with a 10-s anneal gave a doping concentration of $4 \times 10^{20} / \mathrm{cm}^{3}$ near the pillar top, which dropped to around $1 \times 10^{18} / \mathrm{cm}^{3}$ at the edge of the silicide. NiSi work functions (WFs) are known to vary with doping, and in heavily doped silicon, silicidation-induced submonolayer segregation of dopants causes a change in the apparent WF [27]. In low-doped $\mathrm{Si}, \mathrm{NiSi}$ has a WF of around $0.56-0.67 \mathrm{eV}$ [30], [31], whereas, in high-doped silicon, this reduces to a value of around $0.31 \mathrm{eV}$ [30]. This reasoning suggests that silicidation could lead to the formation of a Schottky barrier on the pillar sidewall close to the top S/D junction, which could explain the nonlinear turn on and asymmetry observed in the output characteristic of the silicided v-MOSFET in Fig. 7(a).

To confirm this hypothesis, ATLAS mixed-mode simulations were performed, incorporating numerical models for a Schottky diode and the v-MOSFET. In these simulations, the drain-source current entering the top S/D from the channel sees two conduction paths in parallel [inset in Fig. 11(a)]. One is through the bulk junction and into the NiSi ohmic contact on top of the pillar. The other is along the sidewall surface and into the Schottky NiSi contact on the sidewall of the pillar. The total drain-source current collected at the drain is therefore the sum of these two currents. In simulations, the quantum mechanical tunneling and the thermionic emission models were both turned on. In order to reproduce the measured current drive, arbitrary series resistors (not shown) were introduced into the Schottky and the ohmic conduction paths, and the barrier height was set to a value that reproduced the onset of turn-on in the measured $I_{D S}-V_{D S}$ characteristics.

The simulated output characteristics for both DOT and SOT operations are shown in Fig. 11 for $2-\mathrm{V}$ gate bias. In DOT operation, at low values of $V_{D S}$, the current along the pillar sidewall is limited by the Schottky contact, and hence, the total current is dominated by the path through the bulk junction to the ohmic contact on top of the pillar. However, once the Schottky diode turns on, the current along the pillar sidewall 


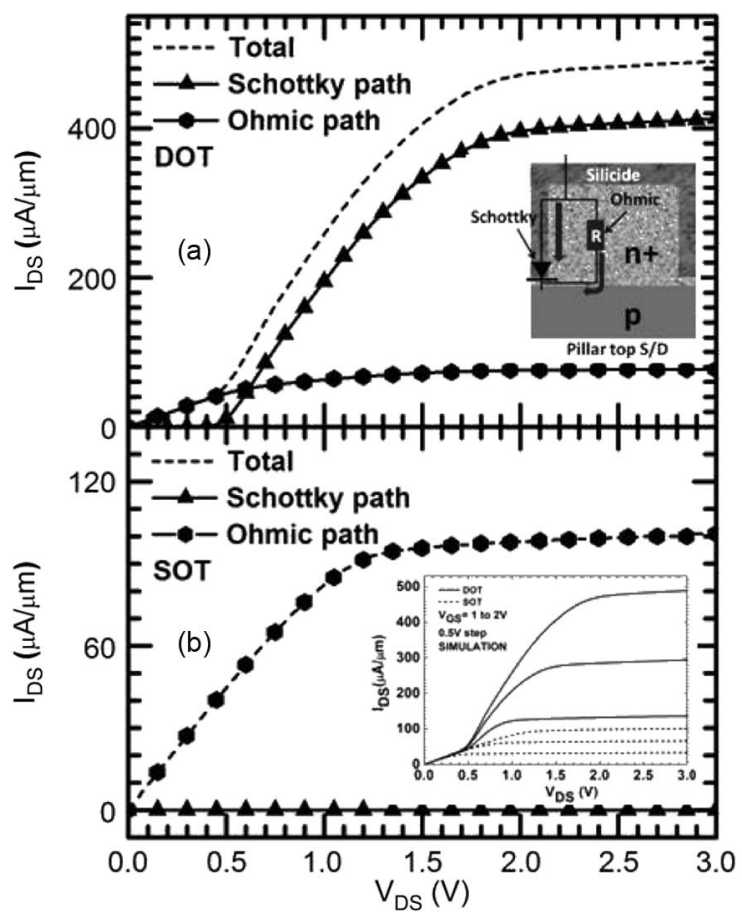

Fig. 11. Simulated output characteristics of the 120-nm silicided surroundgate v-MOSFETs annealed for $10 \mathrm{~s}$ at $1100{ }^{\circ} \mathrm{C}$. (a) DOT operation and (b) SOT operation under a gate bias of $2 \mathrm{~V}$. Results are shown for two current paths through the device, a surface current through a peripheral Schottky contact, and a bulk current to the top ohmic contact. The inset of (b) shows the total current in DOT and SOT operation.

is significantly larger than that through the bulk junction and therefore dominates the total current. In SOT operation, the Schottky diode is reverse biased, so conduction along the pillar surface is completely blocked and the total current is dominated by conduction through the bulk junction. Hence, no nonlinear turn-on is seen at low $V_{D S}$, and the drive current is very low due to a relatively larger resistance expected in the bulk junction conduction path. The total drain-source current in DOT and SOT operation is shown in the inset of Fig. 11(b), and it can be seen that this characteristic successfully reproduces the main features of the measured results in Fig. 7. This analysis confirms that the observed nonlinear turn-on and asymmetrical drive current seen in Fig. 7 are due to the close proximity of the silicidation to the top S/D junction. To characterize the effect of different anneal conditions on this behavior, we have simulated different RTA times using the ATHENA process simulator using measured SIMS profiles to calibrate the simulations. These simulations show that the optimum RTA is around $22 \mathrm{~s}$ at $1100{ }^{\circ} \mathrm{C}$, which places the NiSi interface $35 \mathrm{~nm}$ from the channel and gives a silicidation tip doping density of around $10^{19} / \mathrm{cm}^{3}$, which is sufficient for an ohmic contact.

Finally, we discuss the degradation of the subthreshold slope from 78 to $85 \mathrm{mV} / \mathrm{dec}$ in the silicided 120-nm v-MOSFETs and the general trends of threshold voltage shift and DIBL degradation after silicidation. The small degradation in subthreshold slope suggests the presence of interface states, presumably due to the proximity of the silicided region to the top S/D junction. To further investigate this possibility, we have estimated the increase in the interface state density $\Delta N_{\text {it }}$ and oxide charge $\Delta N_{\text {ot }}$ after silicidation for 80- and 120-nm v-MOSFETs using the change in subthreshold slope and shift of midgap voltage, respectively, [32]. For 80- and 120-nm v-MOSFETs, the values of $\Delta N_{\text {it }}$ are found to be $6 \times 10^{11} / \mathrm{cm}^{2}$ and $1.3 \times 10^{12} / \mathrm{cm}^{2}$, and the values of $\Delta N_{\text {ot }}$ are found to be $2 \times 10^{11} / \mathrm{cm}^{2}$ and $1.9 \times 10^{11} / \mathrm{cm}^{2}$, respectively. The twice higher $\Delta N_{\mathrm{it}}$ in the 120-nm v-MOSFET, compared with the 80-nm v-MOSFET, clearly shows that the closer proximity of the silicidation region to the top S/D junction favors the creation of interface states in the channel. Furthermore, the positive shift of the subthreshold plots in silicided devices indicates that these interface states are acceptorlike, which also agrees with a study of $\mathrm{Ni}$ irradiationinduced modifications of nMOSFETs done by Shinde et al. [33]. A similar reasoning can be used to explain the DIBL degradation after silicidation, as we have discussed in previous work [19].

\section{CONCLUSiON}

We have reported a CMOS-compatible silicidation technology for surround-gate v-MOSFETs, which is integrated with a FILOX process, delivering low-overlap-capacitance and high-drive-current vertical devices. Silicided $80-n m$ vertical nMOSFETs show an excellent drive current improvement in comparison to nonsilicided devices, with improvements of $60 \%$ and $51 \%$ for DOT and SOT operations, respectively. The silicided devices also exhibit an excellent subthreshold slope of $87 \mathrm{mV} / \mathrm{dec}$ and a DIBL of $80 \mathrm{mV} / \mathrm{V}$, compared with $86 \mathrm{mV} / \mathrm{dec}$ and $60 \mathrm{mV} / \mathrm{V}$ for nonsilicided devices. The silicided $80-\mathrm{nm}$ v-MOSFETs have an $f_{T}$ of $20 \mathrm{GHz}$, which is approximately twice the value expected for comparable planar MOSFETs fabricated using the same $0.5-\mu \mathrm{m}$ lithography. This result demonstrates the promise of v-MOSFETs as a route for improving the RF performance of mature CMOS technologies. We have also investigated issues associated with silicidation down the pillar sidewall by reducing the activation anneal time to bring the silicided region closer to the p-n junction at the top of the pillar. In this situation, nonlinear transistor turn-on is observed in DOT operation and dramatically degraded drive current in SOT operation. These results have been explained by the formation a Schottky contact around the pillar perimeter when the silicided region comes too close to the top S/D junction.

\section{ACKNOWLEDGMENT}

The authors would like to thank the Electrum Laboratory, Royal Institute of Technology (KTH), Sweden, for providing facilities for fabrication.

\section{REFERENCES}

[1] M. Masahara, Y. Liu, S. Hosokawa, T. Matsukawa, K. Ishii, H. Tanoue, K. Sakamoto, T. Sekigawa, H. Yamauchi, S. Kanemaru, and E. Suzuki, "Ultrathin channel vertical DG MOSFET fabricated by using ionbombardment-retarded etching," IEEE Trans. Electron Devices, vol. 51, no. 12, pp. 2078-2085, Dec. 2004.

[2] H. Cho, P. Kapur, P. Kalavade, and K. C. Saraswat, "A low-power, highly scalable, vertical double-gate MOSFET using novel processes," IEEE Trans. Electron Devices, vol. 55, no. 2, pp. 632-639, Feb. 2008.

[3] B. Yang, K. D. Buddharaju, S. H. G. Teo, J. Fu, N. Sing, G. Q. Lo, and D. L. Kwong, "CMOS compatible gate-all-around vertical siliconnanowire MOSFETs," in Proc. 38th ESSDERC, 2008, pp. 318-321. 
[4] H. Liu, Z. Xiong, and J. K. O. Sin, "An ultrathin vertical channel MOSFET for sub $100 \mathrm{~nm}$ applications," IEEE Trans. Electron Devices, vol. 50, no. 5, pp. 1322-1327, May 2003.

[5] T. Schulz, W. Rösner, L. Risch, A. Korbel, and U. Langmann, "Shortchannel vertical sidewall MOSFETs," IEEE Trans. Electron Devices, vol. 48, no. 8, pp. 1783-1788, Aug. 2001.

[6] L. Risch, W. H. Krautschneider, F. Hofmann, H. Schäfer, T. Aeugle, and W. Rösner, "Vertical MOS transistor with $70 \mathrm{~nm}$ channel length," IEEE Trans. Electron Devices, vol. 43, no. 3, pp. 1495-1498, Sep. 1996.

[7] H. Gossner, F. Wittmann, I. Eisele, T. Grabolla, and D. Behammer, "Vertical MOS technology with sub-0.1 $\mu$ m channel lengths," Electron. Lett., vol. 31, no. 16, pp. 1394-1396, Aug. 1995.

[8] F. Kaesen, C. Fink, K. G. Anil, W. Hansch, T. Doll, T. Grabolla, H. Schreiber, and I. Eisele, "Optimization of the channel doping profile of vertical sub-100 nm MOSFETs," Thin Solid Films, vol. 336, no. 1/2, pp. 309-312, Dec. 1998.

[9] J. Moers, D. Klaes, A. Tönnesmann, L. Vescan, S. Wickenhäuser, T. Grabolla, M. Marso, P. Kordoš, and H. Lüth, "Vertical p-MOSFETs with gate oxide deposition before selective epitaxial growth," Solid State Electron., vol. 43, no. 3, pp. 529-535, Mar. 1999.

[10] D. Klaes, J. Moers, A. Tönnesmann, S. Wickenhäuser, L. Vescan, M. Marso, T. Grabolla, M. Grimm, and H. Lüth, "Selectively grown vertical Si MOS transistor with reduced overlap capacitances," Thin Solid Films, vol. 336, no. 1/2, pp. 306-308, Dec. 1998.

[11] K. Mori, A. Duong, and W. F. Richardson, "Sub-100 nm vertical MOSFET with threshold voltage adjustment," IEEE Trans. Electron Devices, vol. 49, no. 1, pp. 61-66, Jan. 2002.

[12] E. Gili, T. Uchino, M. M. A. Hakim, C. H. de Groot, O. Buiu, S. Hall, and P. Ashburn, "Shallow junctions on pillar sidewalls for sub- $100 \mathrm{~nm}$ vertical MOSFETs," IEEE Electron Device Lett., vol. 27, no. 8, pp. 692-695, Aug. 2006.

[13] J. M. Hergenrother, D. Monroe, F. P. Klemens, A. Kornblit, G. R. Weber, W. M. Mansfield, M. R. Baker, F. H. Baumann, K. J. Bolan, J. E. Bower, N. A. Ciampa, R. A. Cirelli, J. I. Colonell, D. J. Eaglesham, J. Frackoviak, H. J. Gossmann, M. L. Green, S. J. Hillenius, C. A. King, R. N. Kleiman, W. Y.-C. Lai, J. T.-C. Lee, R. C. Liu, H. L. Maynard, M. D. Morris, S.-H. Oh, C.-S. Pai, C. S. Rafferty, J. M. Rosamilia, T. W. Sorsch, and H.-H. Vuong, "The vertical replacement (VRG) MOSFET: A $50 \mathrm{~nm}$ vertical MOSFET with lithography-independent gate length," in IEDM Tech. Dig., 1999, pp. 75-78.

[14] S. K. Jayanarayanan, S. Dey, J. P. Donnelly, and S. K. Banerjee, "A novel $50 \mathrm{~nm}$ vertical MOSFET with a dielectric pocket," Solid State Electron., vol. 50, no. 5, pp. 897-900, May 2006.

[15] V. D. Kunz, T. Uchino, C. H. de Groot, P. Ashburn, D. C. Donaghy, S. Hall, Y. Wang, and P. L. F. Hemment, "Reduction of parasitic capacitance in vertical MOSFETs by spacer local oxidation," IEEE Trans. Electron Devices, vol. 50, no. 6, pp. 1487-1493, Jun. 2003.

[16] E. Gili, V. D. Kunz, C. H. de Groot, T. Uchino, P. Ashburn, D. C. Donaghy, S. Hall, Y. Wang, and P. L. F. Hemment, "Single, double and surround gate vertical MOSFETs with reduced parasitic capacitance," Solid State Electron., vol. 48, no. 4, pp. 511-519, Apr. 2004.

[17] J. Moers, S. Trellenkamp, A. van der Hart, M. Goryll, S. Mantl, P. Kordoš, and H. Lüth, "Vertical p-channel double gate MOSFETs," in Proc. 33rd ESSDERC, 2003, pp. 143-146.

[18] M. M. A. Hakim, L. Tan, T. Uchino, O. Buiu, W. Redman-White, S. Hall, and P. Ashburn, "Improved sub-threshold slope in RF vertical MOSFETS using a frame gate architecture," in Proc. 38th ESSDERC, 2008, pp. 95-98.

[19] M. M. A. Hakim, L. Tan, O. Buiu, W. Redman-White, S. Hall, and P. Ashburn, "Improved sub-threshold slope in short channel vertical MOSFETS using a FILOX oxidation," Solid State Electron., vol. 53, no. 7, pp. 753-759, Jul. 2009.

[20] M. M. A. Hakim, K. Mallik, C. H. de Groot, W. Redman-White, L. Tan, S. Hall, and P. Ashburn, "A self-aligned silicidation technology for surround-gate vertical MOSFETS," in Proc. 39th ESSDERC, 2009, pp. 363-366.

[21] B. Ricco, P. Olivo, T. N. Nguyen, T.-S. Kuan, and G. Ferriani, "Oxide thickness determination in thin-insulator MOS structures," IEEE Trans. Electron Devices, vol. 35, no. 4, pp. 432-438, Apr. 1988.

[22] C. Spinella, V. Raineri, and S. U. Campisano, "Selective etching of B-doped silicon: Mechanisms and two-dimensional delineation of concentration profiles," J. Electrochem. Soc., vol. 142, no. 5, pp. 1601-1607, May 1995.

[23] N. Arora, MOSFET Models for VLSI Circuit Simulation. New York: Springer-Verlag, 1993.
[24] F. H. De La Moneda, H. N. Kotecha, and M. Shatzkes, "Measurement of MOSFET constants," IEEE Electron Device Lett., vol. EDL-3, no. 1, pp. 10-12, Jan. 1982.

[25] T. H. Lee, The Design of CMOS Radio-Frequency Integrated Circuits., 2nd ed. New York: Cambridge Univ. Press, 2004.

[26] M. Racanelli and P. Kempf, "SiGe BiCMOS Technology for RF circuit applications," IEEE Trans. Electron Devices, vol. 52, no. 7, pp. 12591270, Jul. 2005.

[27] J.-C. Gou, M.-C. Chang, C.-Y. Lu, C. C.-H. Hsu, and S. S.-S. Chung, "Transconductance improvement due to back bias for submicron NMOSFET," IEEE Trans. Electron Devices, vol. 42, no. 2, pp. 288-294, Feb. 1995.

[28] G. A. Sai-Halasz, M. R. Wordeman, D. P. Kern, S. Rishton, and E. Ganin, "High transconductance and velocity overshoot in NMOS devices at the 0.1- $\mu \mathrm{m}$ gate-length level," IEEE Electron Device Lett., vol. 9, no. 9, pp. 464-466, Sep. 1988.

[29] A. Cester, S. Gerardin, A. Tazzoli, and G. Meneghesso, "Electrostatic discharge effects in ultrathin gate oxide MOSFETs," IEEE Trans. Device Mater. Rel., vol. 6, no. 1, pp. 87-94, Mar. 2006.

[30] J. Kedzierski, D. Boyd, P. Ronsheim, S. Zafar, J. Newbury, J. Ott, C. Cabral, Jr, M. Ieong, and W. Haensch, "Threshold voltage control in NiSi-gated MOSFETs through silicidation induced impurity segregation," in IEDM Tech. Dig., 2003, pp. 315-318.

[31] H. Iwai, T. Ohguro, and S.-I. Ohmi, "NiSi technology for scaled CMOS," Microelectron. Eng., vol. 60, no. 1/2, pp. 157-169, Jan. 2002.

[32] P. J. McWhorter and P. S. Winokur, "Simple technique for separating the effects of interface traps and trapped-oxide charge in metal-oxide-semiconductor transistors," Appl. Phys. Lett., vol. 48, no. 2, pp. 133-135, Jan. 1986.

[33] N. Shinde, V. N. Bhoraskar, and S. D. Dhole, "Swift heavy-ion induced trap generation and mixing at $\mathrm{Si} / \mathrm{SiO}_{2}$ interface in depletion n-MOS," Nucl. Instrum. Methods Phys. Res. B, Beam Interact. Mater. At., vol. 242, no. 1/2, pp. 659-662, Jan. 2006.

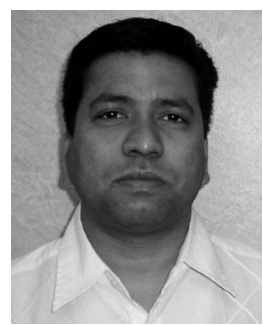

M. M. A. Hakim was born in Dhaka, Bangladesh He received the B.Sc. and M.Sc. degrees in electrical and electronic engineering from Bangladesh University of Engineering and Technology, Dhaka, in 1999 and 2001, respectively, and the Ph.D. degree from the University of Southampton, Southampton, U.K. in 2007. His dissertation topic was low-temperature seeded crystallization of amorphous silicon for transistor in grain technology.

From 1999 to 2008, he was an Assistant Professor with the Department of Electrical and Electronic Engineering, Bangladesh University of Engineering and Technology (BUET), Dhaka, Bangladesh. He is currently a Research Fellow with the Nano Research Group, School of Electronics and Computer Science, University of Southampton. He has interest both for fundamental and applied research in electronics. In the past, he pursued basic research like physics-based simulation of nanodevices, research on material science and fabricated new types of electronic devices. His current research interests include bionanotechnology, next-generation photovoltaics, nonclassical CMOS and beyond CMOS devices, thin-film technology, and process/device simulation.

Prof. Hakim was the recipient of Prestigious Commonwealth Scholarship from 2003 to 2007 .

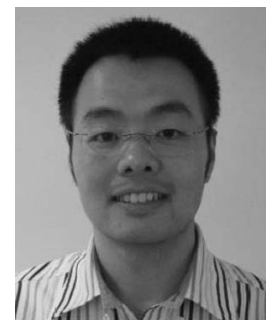

L. Tan received the B.Sc. and Ph.D. degree in electrical engineering from the University of Liverpool, Liverpool, U.K., in 2004 and 2009, respectively. His $\mathrm{Ph} . \mathrm{D}$. thesis was on the feasibility of novel CMOStechnology-compatible vertical MOSFETs for radiofrequency (RF) applications.

He then continued to work on VMOST and passive components for RF applications as a Research Associate. He is currently with the Department of Electrical Engineering and Electronics, University of Liverpool. His research interests include device characterization, compact modeling, and numerical/analytical modeling for underlying physics investigation. 


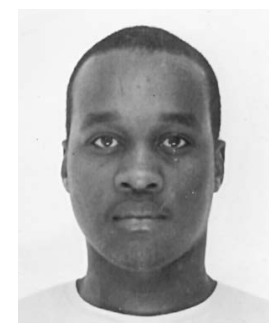

A. Abuelgasim was born in Khartoum, Sudan, in 1985. He received the M.Eng. degree, in 2007, in electronic engineering from the University of Southampton, Southampton, U.K., where he is currently working toward the $\mathrm{Ph} . \mathrm{D}$. degree in the School of Electronics and Computer Science, working on silicon for high-frequency operation with the Nanogroup.

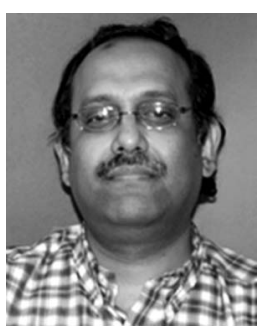

K. Mallik (M'04) received the Ph.D. degree on reduction of dislocations in GaAs by isoelectronic doping from the University of Calcutta, West Bengal, India, in 1998.

He has been with the Raja Ramanna Centre for Advanced Technology, Indore, Government of India; Department of Materials, Oxford University, Oxford, U.K.; and Optoelectronic Research Center, University of Southampton, Southampton, U.K. He is currently a Senior Research Fellow with the School of Electronics and Computer Science, University of Southampton. His research interests are experimental research on electronic and optical properties of materials for device applications, including growth and characterization of semiconductors, oxides, metals, polymers, and nanostructured materials and devices.

S. Connor received the B.Eng. degree in electronics and the Ph.D. degree in solid-state physics from Liverpool University, Liverpool, U.K.

$\mathrm{He}$ is currently involved in managing the Characterization and Modeling Group, Plessy Semiconductors Ltd., Swindon, U.K. This includes the management of both internal and external modeling projects and support activities for new product development and integration. His specialties include noise measurement/modeling and high-frequency compact models. He also has extensive experience of modeling RF passive nonlinear devices (inductors and capacitors) on a number of technologies and providing parasitic models.

A. Bousquet received the B.S. degree in electronics from the University of Science of Montpellier, Montpellier, France, in 1995.

$\mathrm{He}$ is currently a Principal Engineer with Plessey Semiconductors Ltd., Swindon, U.K., and is involved in the development and characterization of high-voltage/high-speed bipolar transistors on silicon-on-insulator technologies. His research interests include CMOS and bipolar device modeling and characterization.

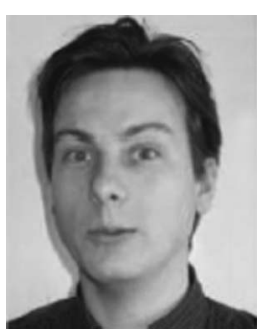

C. H. de Groot (SM'06) received the M.S. degree in physics from the University of Groningen, Groningen, The Netherlands, in 1994 and the $\mathrm{Ph} . \mathrm{D}$. degree from the University of Amsterdam, Amsterdam, The Netherlands, in 1998, with research carried out at the Philips Research Laboratories, Eindhoven, The Netherlands. His Ph.D. dissertation was on $\mathrm{NdFeB}$ permanent magnets and magnetism in rare-earth intermetallic compounds.

From 1998 to 2000, he was a Research Fellow with the Francis Bitter Magnet Laboratory, Massachusetts Institute of Technology, Cambridge, where he conducted a research on spin tunnel junction. Since 2000, he has been with the School of Electronics and Computer Science, University of Southampton, Southampton, U.K., where he is currently an Associate Professor. His research interest includes nanoelectronics, with emphasis on spintronics and post-CMOS field-effect transistors.

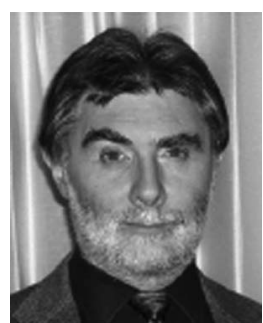

W. Redman-White (M'83-SM'08) received the M.Sc. and Ph.D. degrees from Southampton University, Southampton, U.K., in 1980 and 1983 respectively.

Since 1990, he has been with NXP (formerly Philips Semiconductors), Southampton, U.K., where he is currently a Fellow. He has also been in San Jose, CA, and Caen, France, working on optical storage, wireless local area networks, cellular radio, Bluetooth, digital audio, TV, satellite baseband, high-speed serial links, and car security. He was previously with Motorola, Geneva, GEC-Marconi Research London, and Post Office Telecommunications, London. Concurrently with his industrial activities, he has also been a faculty position in School of Electronics and Computer Science, University of Southampton, Southampton, U.K., since 1983, and is currently a Full Professor of integrated circuit design. He has published 120 papers. He is the holder of more than 12 patents, with several still pending. His research and teaching is centered on analog and RF IC design, and design issues in SOI CMOS technology.

Dr. Redman-White served as Associate Editor for the IEEE JOURNAL OF Solid-STATE Circuits from 1996 to 2002. He is currently the Analog SubCommittee Chair for the IEEE International Solid State Circuits Conference, has twice been Technical Program Chair of the European Solid State Circuits Conference (1997 and 2008), and is a member of the steering committee for the European Solid State Circuits and Devices Conference series (ESSCIRC/ESSDERC).

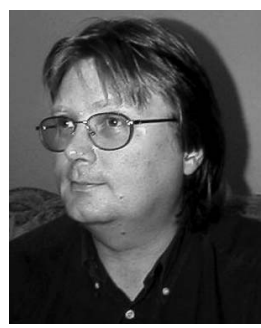

S. Hall (M'93-SM'07) received the Ph.D. degree from the University of Liverpool, U.K., in 1987, for his research on a new form of integrated injection logic in the GaAs/AlGaAs materials system.

$\mathrm{He}$ is currently with the Department of Electrical Engineering and Electronics, University of Liverpool, Liverpool, U.K, where he was Head of the Department from 2001 to 2009 . He has published more than 200 conference and journal papers in the area of silicon technology, devices, and circuits. These include novel measurements and contributions to the understanding of MOS-related interfaces and materials quality. He has successfully designed and built novel MOS and bipolar devices in silicon for about 20 years. More recently, his work encompasses high- $k$ dielectrics, novel devices, and gate-level circuits relating to micropower and biologically inspired concepts. His research interests include material characterization, device physics, and innovative device design and gate-level circuits.

Dr. Hall was the Technical Program Chair of the European Solid-State Device Research Conference (ESSDERC) 2008 and is currently serving on the Steering Committee of ESSDERC/European Solid-State Circuits Conference (ESSCIRC) and Insulating Films on Semiconductors (INFOS) Conference, for which he was Vice-Chair in 2009 and is a member of the Steering Committee from 2009 .

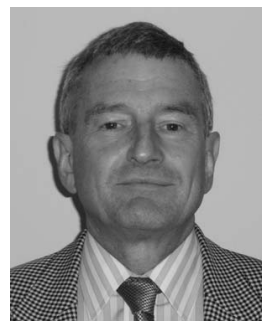

P. Ashburn (M'89) was born in Rotherham, U.K. in 1950. He received the B.Sc. degree in electrical and electronic engineering and the Ph.D. degree from the University of Leeds, Leeds, in 1971 and 1974, respectively. His dissertation topic was an experimental and theoretical study of radiation damage in silicon p-n junctions.

In 1974, he joined the technical staff of Philips Research Laboratories and initially worked on ionimplanted integrated circuit bipolar transistors and then on electron lithography for submicrometer integrated circuits. In 1978, he joined the academic staff of the School of Electronics and Computer Science, University of Southampton, Southampton, as a Lecturer, and is currently is the holder of a Personal Chair in Nanoelectronics. Since taking up a post at Southampton University, he has worked on a variety of silicon-based RF device technologies. He has authored or coauthored 280 papers in the technical literature, given many invited papers on RF devices and technology, and authored books on the Design and Realization of Bipolar Transistors in 1988 and on Silicon Germanium Heterojunction Bipolar Transistors in 2003. He has undertaken collaborative and consultancy work for many U.K. and international companies, and has served as a nonexecutive director of Innos Ltd. He is currently the Director of the Southampton Nanofabrication Centre, which is a $£ 100$ million state-of-the-art facility for semiconductor nanofabrication and characterization. His current research interests include RF vertical MOSFETs, CMOS-compatible carbon nanotubes, and silicon nanowire biosensors. 